\title{
Electrophoretic Versus Dielectrophoretic Nanoparticle Patterning Using Optoelectronic Tweezers
}

\author{
Juan F. Muñoz-Martínez \\ Departamento de Mecánica de Fluidos y Propulsión Aeroespacial, ETSIAE, \\ Universidad Politécnica de Madrid, 3 Plaza Cardenal Cisneros, 28040 Madrid, Spain \\ and Departamento de Física de Materiales, Facultad de Ciencias, Universidad Autónoma de Madrid, \\ 7 Francisco Tomás y Valiente, 28049 Cantoblanco, Madrid, Spain \\ José B. Ramiro and Ángel Alcázar \\ Departamento de Mecánica de Fluidos y Propulsión Aeroespacial, ETSIAE, \\ Universidad Politécnica de Madrid, 3 Plaza Cardenal Cisneros, 28040 Madrid, Spain \\ Ángel García-Cabañes and Mercedes Carrascosa* \\ Departamento de Física de Materiales, Facultad de Ciencias, Universidad Autónoma de Madrid, \\ 7 Francisco Tomás y Valiente, 28049 Cantoblanco, Madrid, Spain \\ (Received 1 February 2017; revised manuscript received 8 May 2017; published 26 June 2017)
}

\begin{abstract}
Currently, there is increasing interest from many scientific disciplines in the development of systems that are able to sort and arrange many objects in parallel at the nano- and micrometric scale. Among others, photovoltaic tweezers (PVT) are an optoelectronic technique for trapping and patterning nano- and microobjects in accordance with an arbitrary light profile. In this work, the differential features of electro- and dielectrophoretic (EP and DEP) nanoparticle (NP) patterning using PVT are deeply investigated. The study is carried out through theory and experiments. The developed theory extends the applicability of a previously reported model to be able to compute EP potentials and to obtain numerical values for the EP and DEP potential energies. Two-dimensional patterns of charged and neutral aluminum NPs are fabricated on top of $\mathrm{Fe}: \mathrm{LiNbO}_{3}$ crystals, and different light distributions and other experimental parameters (crystal thickness and NP concentration) are compared. Patterns of charged and neutral NPs show remarkable differences in both particle density distribution and fidelity to the original light profile. The observed different features between EP and DEP trapping are satisfactorily explained by the theoretical analysis. The results provide routes for the optimization of the NP arrangements for both regimes.
\end{abstract}

DOI: 10.1103/PhysRevApplied.7.064027

\section{INTRODUCTION}

Manipulation and patterning of nano- and micro-objects is a fundamental issue in many, very active fields such as nanotechnology and biotechnology [1-6]. To this end, different purely optical [7] or optoelectric techniques [2] have been developed. The former ones (optical tweezers) are more suitable for single-particle manipulation, whereas the latter methods allow parallel manipulation of many objects. Among other optoelectronic techniques, photovoltaic tweezers (PVT) are an emerging, flexible, low-cost technique that allows massive and parallel particle trapping and patterning on the surface of certain ferroelectric materials [8,9]. This tool is based on the remarkably high light-induced electric fields generated in those materials via a singular phenomenon, the so-called bulk photovoltaic (PV) field. When such ferroelectric crystals are illuminated, electrons are photoexcited along a preferential direction (the polar or $c$ axis) giving rise to a PV

\footnotetext{
*Corresponding author. m.carrascosa@uam.es
}

electronic current. This PV current and the subsequent charge migration and redistribution are responsible for the high PV electric fields inside the material. For the most common ferroelectric material used as PV substrate, Fe-doped $\mathrm{LiNbO}_{3}$, the PV fields can reach values as high as $\left[10^{4}-\right.$ $\left.\left(2 \times 10^{5}\right)\right] \mathrm{V} / \mathrm{cm}[10,11]$. These fields extend outside the crystal and attract its surface charged or neutral particles existing in its surroundings through either electrophoretic (EP) or dielectrophoretic (DEP) forces, respectively [8].

Recently, PVT has attracted considerable interest due to its flexibility and easy operation. Unlike optical tweezers, this technique allows the simultaneous manipulation of a large number of particles and efficiently operates at low or moderate intensities [9]. Among its advantages over other optoelectronic techniques, PVT do not require any electrode or voltage sources, as electric fields are generated simply by illumination. In addition, PV electric fields remain in the material for months or even years under appropriate dark conditions, favoring long pattern stability [8]. These characteristics together with its efficiency and low cost are making PVT a competitive tool for organic and inorganic particle 
manipulation. This technique has been successfully applied in different fields including (i) microfluidics for microdroplet dynamic deformation, patterning, and splitting [12-14] and particle desorption mass spectrometry microchannel fabrication [15], (ii) plasmonics, as a tool for obtaining plasmonic platforms for fluorescence enhancement of organic and biomolecules [16], (iii) biophotonics and biomedicine as a method of killing cancer cells [17] and for bacteria, pollen, and spore arranging [18,19], and (iv) photonics, for the fabrication of flexible diffractive optical elements [20].

Although PVT are able to manipulate both charged and neutral particles, all theoretical calculations are focused on the patterning of neutral particles [21]. Furthermore, most reported experiments on the trapping of nano- $[22,23]$ and micro-objects [24-27] deal with different illumination and crystal configurations but considering only particles with no electric charge. Concerning electrophoretic trapping, a few experimental results have been reported $[28,29]$. The information available on the specific features of EP particle trapping and on the comparison with the DEP regime is still scarce. A comparative analysis of this issue is interesting not only as a basic research topic but as an important question to be answered in order to extend the possibilities of the PVT technique to new, naturally electrically charged targets such as DNA and other biological or inorganic entities [30]. Also, the different expected features of EP and DEP trapping increase the versatility and possibilities of the PVT technique when using objects that can be either charged or neutral, as the metallic NP used in the present work.

The aim of this work is to delve into the main features of EP and DEP trapping and the resulting NP patterns and to compare both configurations. To this end, the present study is performed via theoretical simulations and experiments. In Sec. II, we address a series of calculations of EP and DEP potential energies in the vicinity of a photovoltaic crystal. These calculations are developed applying, and extending when needed, a previously reported model for DEP trapping [31]. In Sec. III, we present a set of patterning experiments on $\mathrm{Fe}: \mathrm{LiNbO}_{3}$ with both charged and neutral aluminum nanoparticles and analyze them with the help of the theoretical potential energy curves. Finally, we deduce the advantages, disadvantages, and main features of each configuration, paying special attention to the fidelity of the particle pattern to the illumination profile.

\section{THEORETICAL MODEL}

For ease of understanding, let us briefly describe a few more details of the PVT before introducing the theoretical model itself. Particle trapping by PVT is carried out in two stages: (i) sample illumination and (ii) particle approach to the crystal and trapping onto its surface. These stages can be simultaneous or consecutive. In this work, the consecutive method is used, as we find that it allows better control of the experimental parameters and reproducibility. A diagram of this consecutive procedure is shown in Fig. 1. In the first step

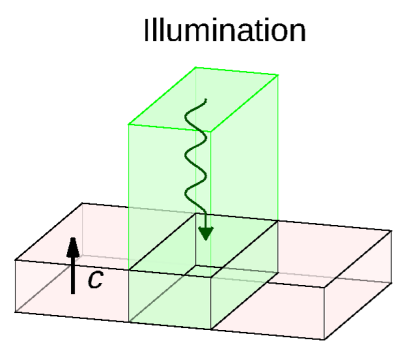

(a)

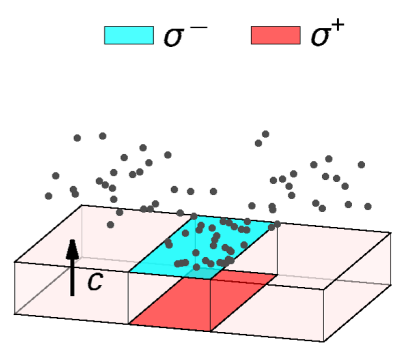

(b)
FIG. 1. Diagram of the PVT technique. (a) Illumination of a $z$-cut crystal with a homogeneous light distribution. (b) Particle trapping due to the space-charge distribution and the associated external electric field.

[Fig. 1(a)], a ferroelectric crystal sample is illuminated with the desired light pattern, and, due to the photovoltaic effect, an electron current is established in the material. As a consequence, a charge redistribution occurs inside the crystal, generating a space-charge distribution in the bulk and the related electric fields inside and outside (evanescent field) the sample. In this work, to carry out the second stage [Fig. 1(b)], the crystal is introduced in a suspension of the particles to be trapped. Depending on the charge state of the particles, the evanescent field gives rise to EP or DEP forces on them, which arrange and trap the objects on the crystal surface. The orientation of the $c$ axis in the substrate is a key parameter for the PVT, and it has been well studied elsewhere [27], as it is the main factor that determines the different patterning capabilities of $x$-cut ( $c$ axis parallel to the surface) and $z$-cut (normal to the surface) crystals. Regarding this aspect, on the one hand, we preferentially use $z$-cut samples since they allow 2D patterning faithful to the illumination, whereas the $x$-cut ones do not [27,31]. On the other hand, we show how $x$-cut samples have turned out to be a good configuration for testing the charge state of particles.

As can be deduced from above, the evanescent electric field in the proximity of the surface of the photovoltaic crystal is a key parameter for PVT operation. Because of the complexity of this electric field, a theoretical model that is able to describe and predict it under specific experimental conditions is necessary. As we mention in the Introduction, the theoretical simulations presented in this work are based on a previously published model for DEP trapping [31]. The cited model is a simplified approach to the steady-state solution of the Kukhtarev equations [32], the system of partial differential equations that describes the charge transport and redistribution inside a photovoltaic crystal, as $\mathrm{Fe}: \mathrm{LiNbO}_{3}$, for moderate light intensities. This simplified model focuses on the current-density evolution:

$$
\boldsymbol{J}=q \mu n \boldsymbol{E}-q D \nabla n+q s I N_{D} L_{\mathrm{PV}} \hat{\boldsymbol{u}}_{\mathrm{PV}} .
$$

In Eq. (1), $\boldsymbol{J}$ is the current density, $\mu$ the electron mobility, $\boldsymbol{E}$ the total electric field acting on the crystal, $D$ the diffusion coefficient, $s$ the photoionization cross 
section, $I$ the local light intensity, $N_{D}$ the donor concentration, $L_{\mathrm{PV}}$ the photovoltaic transport length, $\hat{\boldsymbol{u}}_{\mathrm{PV}}$ a unit vector in the direction of the polar axis, $n$ the free-carrier concentration, and $q$ the elementary charge. In the model, the diffusion term $(q D \nabla n)$ is disregarded, as it is much lower than the other two (drift and photovoltaic) in typical experimental conditions. This way, the steady-state electric field $\left(\boldsymbol{E}_{\text {sat }}\right)$ reached in the center of an isolated $\mathrm{Fe}: \mathrm{LiNbO}_{3}$ cuboid homogeneously illuminated [see Fig. 2(a)] can be easily calculated taking into account that at steady state the current density is null, so that $\boldsymbol{E}_{\text {sat }}$ becomes

$$
\boldsymbol{E}_{\mathrm{sat}}=-\frac{s I N_{D} L_{\mathrm{PV}}}{\mu n} \hat{\boldsymbol{u}}_{\mathrm{PV}}
$$

As the carrier concentration is proportional to the local light intensity, $\boldsymbol{E}_{\text {sat }}$ is dependent only on the crystal properties. Once $\boldsymbol{E}_{\text {sat }}$ is calculated, the model allows us to obtain the saturation space-charge density generated at the faces of the $\mathrm{Fe}: \mathrm{LiNbO}_{3}$ cuboid (see Fig. 2). In this model, the charge density is homogeneous. From this charge distribution, the evanescent (fringe) electric field $\left(\boldsymbol{E}_{\text {ext }}\right)$ is numerically calculated. Furthermore, using the dipole approximation for neutral isotropic particles, one can obtain the DEP force $[31,33]$ that is the gradient of the dielectrophoretic potential energy:

$$
\begin{aligned}
& \boldsymbol{F}_{\mathrm{DEP}}=\boldsymbol{\nabla}(\boldsymbol{p} \cdot \boldsymbol{E})=\boldsymbol{\nabla}\left(\alpha E_{\mathrm{ext}}^{2}\right), \\
& U_{\mathrm{DEP}}=-\alpha E_{\mathrm{ext}}^{2},
\end{aligned}
$$

where $\alpha$ is the scalar particle polarizability and depends on the particle's own properties and its surrounding medium [33]:

$$
\alpha=2 \pi r^{3} \varepsilon_{0} \varepsilon_{m} \frac{\varepsilon_{p}-\varepsilon_{m}}{\varepsilon_{p}-2 \varepsilon_{m}} .
$$

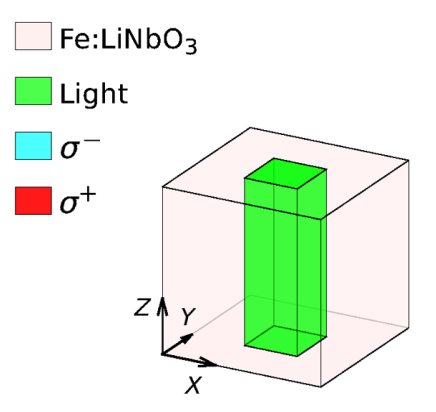

(a)

(c)

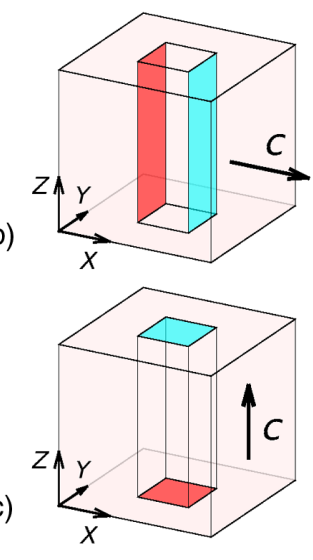

FIG. 2. Illumination diagram of a single cuboid (a) and chargedensity distribution inside the photovoltaic crystal in the (b) $x$ cut and (c) $z$ cut.
In Eq. (5), $r$ stands for the radius of the particle, $\varepsilon_{0}$ for the vacuum permitivity and $\varepsilon_{m}$ and $\varepsilon_{p}$ for the medium and particle relative permitivities, respectively. For metallic nanoparticles in typical dispersive media $\left(\varepsilon_{p} \gg \varepsilon_{m}\right)$ like the ones used in Sec. III, the previous equation can be simplified to

$$
\alpha=2 \pi r^{3} \varepsilon_{0} \varepsilon_{m} .
$$

In order to compare the EP and DEP regimes, in this work we extend the applicability of the theoretical model that we describe above to be able to compute not only the DEP potential energy but also the EP one associated to the same space-charge-density distribution of a crystal. This EP potential energy can be obtained directly from the well-known expression

$$
U_{\mathrm{EP}}=-\int_{l} Q \boldsymbol{E}_{\mathrm{ext}} \cdot d \boldsymbol{l},
$$

where $Q$ is the particle electric charge, and $l$ is an arbitrary curve that connects a point where the potential energy is known to the point $P_{i}$ where this value is wanted. For the sake of simplicity, zero potential energy is fixed at infinity, and our integration path is a line perpendicular to the crystal surface. Thus, the integral of expression (7) turns into

$$
U_{\mathrm{EP}}\left(X_{i}, Y_{i}, Z_{i}\right)=-\int_{\infty}^{Z_{i}} Q E_{\mathrm{ext}, z}\left(X_{i}, Y_{i}, z\right) d z
$$

in which $Z_{i}$ is the height above the crystal where the potential is calculated. We assume the same spatial discretization and expressions of the external electric field as those obtained in Ref. [31].

The potential energy functions $U_{\mathrm{DEP}}$ and $U_{\mathrm{EP}}$ are the main tools to predict and analyze the main features of the patterns since NPs tend to trap in its minima. It should be noted that the model does not take into account the interaction between different cuboids, and it works only with light and dark illumination profiles. Therefore, in principle, it is limited to describe pixelated illumination geometries compounded by many cuboids. Although a more complex model that overcomes these limitations has been already published by the authors [21], it is valid only for an $x$-cut configuration, and it can be used only to check $x$-cut results. Because of the greater complexity presented by the $z$ cut for theoretical modeling, a similar detailed model cannot be found in the literature. In addition, the present model does not take into account particle-particle interaction. The presence of a particle trapped on the surface of the substrate locally modifies the electric field and so the effective potential felt by the next one. The lower the period, the more important this effect [25]. Nevertheless, we show that the simple model presented above is good enough to reveal the most important features of EP and DEP trapping. 


\section{A. Theoretical results and analysis}

The model is applied to simulate EP and DEP potential energy curves for a $250-\mu \mathrm{m}$-side single square homogeneous light distribution, similar to the one shown in Fig. 2. The results are presented in Fig. 3 for the $x$ - [Figs. 3(a) and 3(c)] and $z$-cut [Figs. 3(b) and 3(d)] crystals at different distances from their surfaces. In order to obtain quantitative values for the potential energies, we use the properties of the particles used in Sec. III $(\varnothing=70 \mathrm{~nm}$ aluminum NP in hexane; see Table I). Because of the difficulties of measuring the total charge of a NP suspended in a nonpolar medium, a minimum positive charge density is estimated for the calculation of EP potential energies, as we explain below. First, the peak DEP force is obtained from the DEP potential energy curves in the $z$ cut, resulting in around $3 \mathrm{nN}$ at $1 \mu \mathrm{m}$ from the crystal surface. Then, the minimum total charge of a single NP is estimated by making the EP force peak 1 order of magnitude larger than the DEP one under the same conditions. This way, we ensure that DEP forces can be disregarded as charged particles. The obtained minimum charge, around $2 \times 10^{-17} \mathrm{C}$, is in good agreement with the previous calculations made for similar theoretical arrangements by Mokrý et al. [34]. According to Table II, it is evident that for both EP and DEP regimes, the potential energy values are several orders of magnitude larger than the thermal energy at room temperature $k T$, being $k$ the Boltzmann constant and $T$ the absolute temperature.

Starting with the $x$ cut, this square-type illumination generates two opposite-charged planes inside the crystal [see Fig. 2(b)]. We show that while the dielectrophoretic potential energy (proportional to $E^{2}$ ) is symmetric [Fig. 3(c)], the electrophoretic one is clearly antisymmetric [Fig. 3(a)]. The DEP potential energy is also sharper, and its
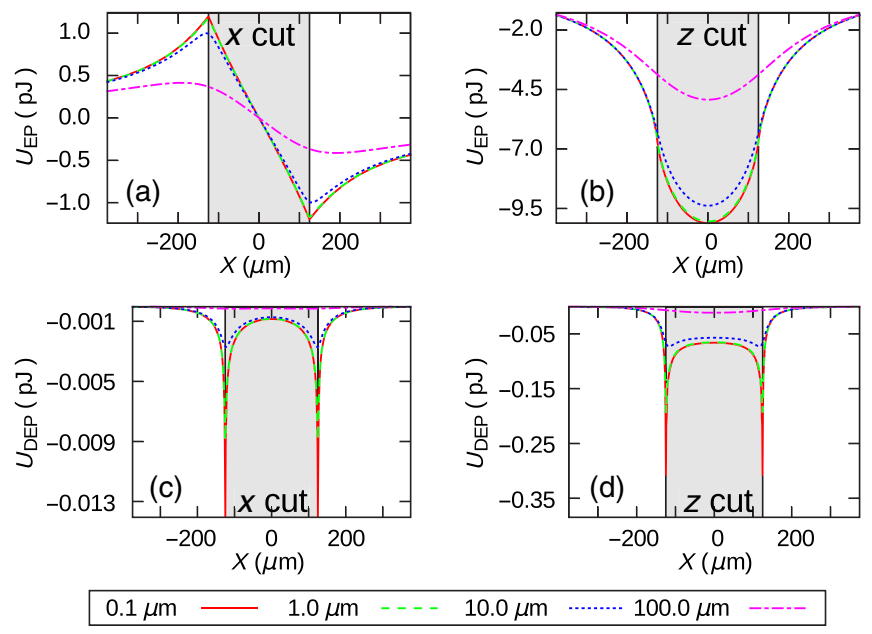

FIG. 3. EP (a),(b) and DEP (c),(d) potential energies at four distances from the surface due to a single square illumination in the $x$ cut (a),(c) and $z$ cut (b),(d). Potential energies are calculated at the midline. In the $z$ cut, they are calculated on the $c+$ face of the crystal.
TABLE I. Experimental parameters used in the simulations.

\begin{tabular}{lcc}
\hline \hline & Particles & Medium \\
\cline { 2 - 3 } Material & Aluminum & Hexane \\
\hline Relative permitivity & $\infty$ & $1.88(\text { at } 300 \mathrm{~K})^{\mathrm{a}}$ \\
Surface-charge density $\left(\mathrm{mC} / \mathrm{m}^{2}\right)$ & 0.32 & $\ldots$ \\
Diameter $(\mathrm{nm})$ & 70 & $\cdots$ \\
\hline \hline
\end{tabular}

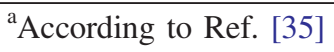

TABLE II. Maximum absolute values of the potential energies shown in Fig. 4 and thermal energy.

\begin{tabular}{lcc}
\hline \hline & $x$ cut & $z$ cut \\
\hline EP $(\mathrm{pJ})$ & 1.19 & 10.12 \\
DEP $(\mathrm{pJ})$ & $1.40 \times 10^{-2}$ & $3.85 \times 10^{-1}$ \\
Thermal at $300 \mathrm{~K}(\mathrm{pJ})$ & \multicolumn{2}{c}{$4.14 \times 10^{-9}$} \\
\hline \hline
\end{tabular}

minima are extremely defined for close distances to the surface. Then, neutral particles should arrange in two identical particle strips, whereas charged particles are expected to trap only on one of the edges of the illuminated area depending on its sign.

In the case of the $z$ cut, EP [Fig. 3(b)] and DEP [Fig. 3(d)] potential energies are calculated at the $c+$ face of the crystal, where the charge density is negative. The potential wells are both symmetrical, in accordance with the fact that the pattern itself is symmetric and electron migration inside the crystal is perpendicular to its surface. However, for the EP case, the potential energy softly varies from far away from the illuminated region to a minimum at the center, whereas for the DEP regime, there is a strong edge effect at the boundary of the illumination zone. This latter case presents very deep wells close to the surface corresponding to the edges of the square and almost a constant higher value in the center [Fig. 3(d)]. From these potential energies, one expects a main difference between the EP and DEP particle profiles in the $z$ cut: for neutral particles, one should expect them to distribute through the whole illuminated spot with preferential trapping at the borderline of the light profile, while charged ones are expected to place mostly in the center of the illuminated region and fade towards the edges.

To sum up, the theoretical model shows clear differences in EP and DEP potential energies for a single square illumination in both cuts. These differences are expected to be translated in diverse patterning features depending on each crystal cut and particle charge state and to be present somehow in more complex trapping profiles.

\section{EXPERIMENTAL PROCEDURE}

As we mention in the previous section, nanoparticle patterns are obtained in a two-step process. First, the crystal sample is illuminated, and then it is introduced in the particle 
suspension. As PV substrates, 1-mm-thick $x$ - and $z$-cut $\mathrm{Fe}: \mathrm{LiNbO}_{3}$ crystals with $0.1-\mathrm{wt} \%$ iron impurities are used. The suspensions contain aluminum nanoparticles $(\varnothing=70 \mathrm{~nm})$ in hexane. As the aim of this work is the comparison of EP and DEP trapping, different pairs of suspensions with the same nanoparticle concentration are made; one of each pair has neutral nanoparticles, and the other one has positively charged ones. The $\mathrm{NP}$ concentrations are between 0.01 and $0.2 \mathrm{~g} / \mathrm{L}$ $\left[\left(\left[2 \times 10^{13}\right)-\left(4 \times 10^{14}\right)\right] \mathrm{NP} / \mathrm{L}\right]$. Except for two cases explicitly indicated in the text, all other experiments are conducted with NP concentrations of $0.04 \mathrm{~g} / \mathrm{L}$ $\left(\left(8 \times 10^{13} \mathrm{NP} / \mathrm{L}\right)\right)$. The immersion times are about $40 \mathrm{~s}$. For crystal illumination, a frequency-doubled Nd:YAG laser at $532 \mathrm{~nm}$ is used. The beam is shaped by a spatial light modulator (SLM) model HOLOEYE LC-R 1080 together with a lens and a pair of crossed polarizers. According to the setup provided by the manufacturer, the laser beam is first polarized and then reflected on the SLM screen, where the desired light profile is presented. The different diffraction orders of the outcoming beam are collected by the lens, and the original image is reconstructed on its image plane. The phase changes induced in the light beam by the SLM are turned into amplitude modulation when it propagates through the second polarizer. Light intensities on the sample are approximately $10 \mathrm{~W} / \mathrm{m}^{2}$, and the illumination time is $10 \mathrm{~min}$.

In order to check the electric charge of the Al NP during the study, a control test based on the potential energy curves shown in Figs. 3(a) and 3(c) is developed. Before and after each experiment, we illuminate an $x$-cut sample with a homogeneous single-slit pattern and trap on it the particles from the suspension we want to test. According to the EP and DEP curves of Figs. 3(a) and 3(c), one should expect a single strip of particles if the NPs are charged and two if they are not. An example of this illustrative test is shown in Fig. 4. If one compares the experimental patterns with the theoretical potential energy curves of Figs. 3(a) and 3(c), one can deduce that in Fig. 4(a), most particles have electric charge, while in Fig. 4(b), they are neutral. Making a good calibration, this experiment can be used to estimate quantitatively the

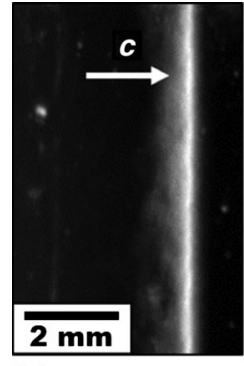

(a)

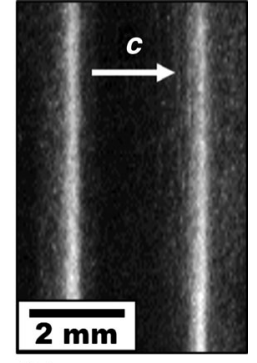

(b)
FIG. 4. Aluminum nanoparticles deposited on the surface of an $x$-cut $\mathrm{Fe}: \mathrm{LiNbO}_{3}$ crystal after single-slit illumination. Positively charged (a) and electrically neutral (b) aluminum nanoparticles. proportion of charged and uncharged particles in a suspension. Even more, the charge sign can be determined if the positive and negative ends of the polar axis are located and vice versa. Therefore, the $x$-cut configuration is a simple and efficient way to check the charge state of the NP.

\section{A. Experimental results and discussion}

In order to deeply investigate the differences between the EP and DEP patterns, experiments using z-cut substrates are preferred, as this cut is able to successfully reproduce 2D light patterns, whereas an $x$ cut is not $[9,27]$. To this end, we develop a series of experiments of different light profiles and experimental parameters. The first light profile we study is represented in Fig. 5(a). It consists of a $7 \times 9$ checkered pattern, and each square has $250-\mu \mathrm{m}$ sides.

In Fig. 5, the patterns made with charged [Fig. 5(b)] and neutral [Fig. 5(c)] aluminum NP particles are shown. In the case of positively charged particles, there is a clear radial

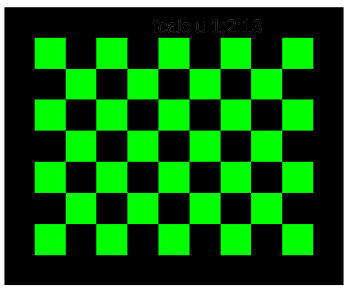

(a)

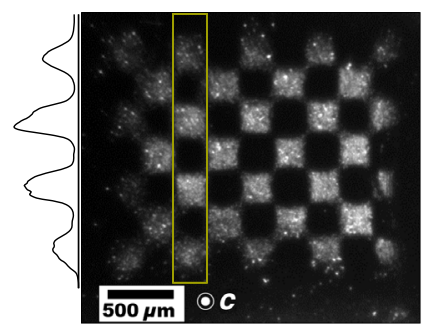

(b)

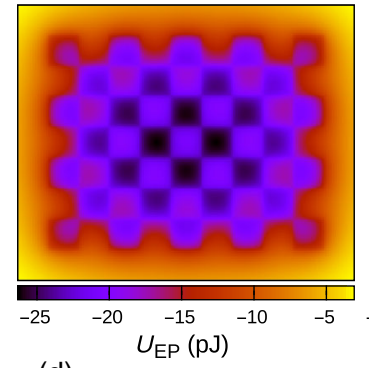

(d)

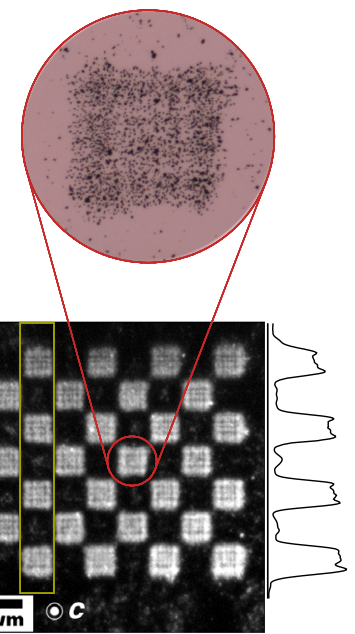

(c)

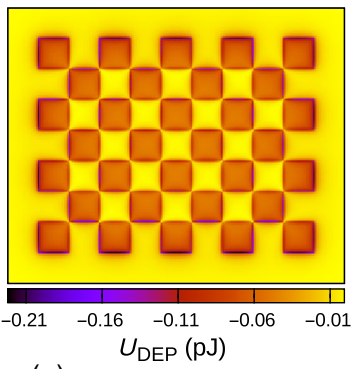

(e)
FIG. 5. Checkered pattern, 250- $\mu \mathrm{m}$ square sides. (a) Light profile. (b),(c) Charged and neutral aluminum nanoparticle patterns on $z$-cut $\mathrm{Fe}: \mathrm{LiNbO}_{3}$ and the corresponding particle density profiles through the highlighted column. (d),(e) EP and DEP potential energies at $1 \mu \mathrm{m}$ from the crystal surface. Dark and light colors indicate low and high values of the potential energies, respectively. 
particle density gradient from the edges towards the center: the squares on the corners are barely defined, while the inner ones are complete, although they are a little blurry and smaller than the ones that form the illumination. On the contrary, when the particles are neutral, all the squares are basically equal and very well defined. In fact, there is higher particle density at the square sides, as can be clearly seen in the inset. The particle density profiles added in Figs. 5(b) and 5(c) are made for both patterns through the third column of squares, and they confirm these differences (see the Fig. 5 caption). The one corresponding to the EP trapping shows a higher NP density of the inner squares and the smooth variation of this parameter inside each one individually. On the contrary, the DEP particle density has steep boundaries between the particle and no-particle regions and larger particle trapping at the square edges.

The charged and neutral NP patterns are very different, as they are expected to be in light of the Sec. II analysis. In order to better explain these different features, we make additional theoretical calculations of the EP [Fig. 5(d)] and DEP [Fig. 5(e)] potential energies using the same parameters as those of the experiments. They are calculated at $1 \mu \mathrm{m}$ from the crystal surface. In the plots shown in Fig. 5, the cool colors indicate low values of the potential energy and the warm colors high ones. For obtaining numerical values, the same assumptions as for Fig. 3 are made. A clear agreement with the experimental patterns described above is found. The global EP potential energy softly varies from the edges of the illumination towards the center. This characteristic matches with the fading of the particle density and the definition observed in the experimental pattern [Fig. 5(b)]. In the DEP potential, all the squares are mostly equal, sharply defined, although if one pays attention to the sides of every single square, a line of much lower potential energy is visible in purple (it is best noticeable in the border squares). This narrow region of extremely low potential is the responsible of the larger number of particles in the edges of the squares shown in Fig. 5(c).

It is worthwhile to also look at some particular pattern details of the EP and DEP trapping. Looking again at Figs. 5(b) and 5(c), a faint NP trapping is visible in the center of the nonilluminated areas in the DEP, whereas in the $\mathrm{EP}$, the regions between the squares are basically clean [this effect also appears in the particle density profiles of Fig. 5(c)]. The explanation can be found in Figs. 3(b) and 3(d). Far away from the crystal surface, both potential energy curves are almost flat, which makes the particles distribute homogeneously at $1-10 \mu \mathrm{m}$ above the crystal. Closer to its surface, both are lower-as they are attractivebut while the EP graph shows a soft decrease towards the center of the square, the DEP potential remains essentially flat outside the illuminated square. From this potential distribution we conclude that neutral particles will feel lower lateral forces than charged ones, so they have a larger probability to be trapped in nonilluminated areas.

In order to ensure that the effects described above are not specific to the chosen pattern but characteristic of EP and DEP trapping, we test other simple geometries with different symmetries, i.e., the periodic pattern of circles shown in Fig. 6. In this pattern, each circle is $250 \mu \mathrm{m}$ in diameter, as it is the square's side length. The same $z$-cut $\mathrm{Fe}: \mathrm{LiNbO}_{3}$ crystals and aluminum NP as in the previous experiment are used. Again, we address the experimental patterns together with the theoretical simulations. The particle patterns are similar to those presented in Fig. 5, although the edge effects are less noticeable, probably because of the radial symmetry of the motives. Nevertheless, there is still a soft increase of the particle density and definition towards the center in the EP trapping [Fig. 6(a)], and in many of the circles of the DEP pattern [Fig. 6(b)], their edges have a larger number of NPs than the inside (top left). In addition, although the light pattern is exactly the same in both cases, the charged particles seem to generate smaller circles due to the global envelope and the gentle slope of the potential wells.

Therefore, the EP potential energy curve is smoother than the DEP one, as shown in Fig. 3. In fact, the main features we predict for a single square in each kind of potential also appear as an envelope in a more complex pattern, which is translated into two very different kinds of patterns depending on the charge state of the particles. If the particles are charged, one can expect a gradient in both trapped particle density and pattern definition from the edges of the pattern towards the center. In the case of neutral particles, the definition of the whole pattern should

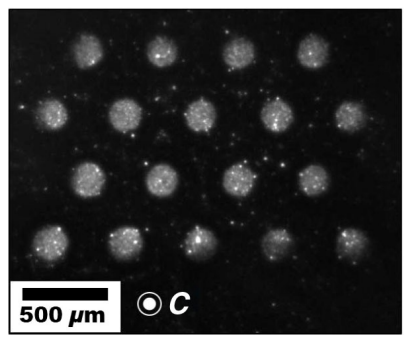

(a)

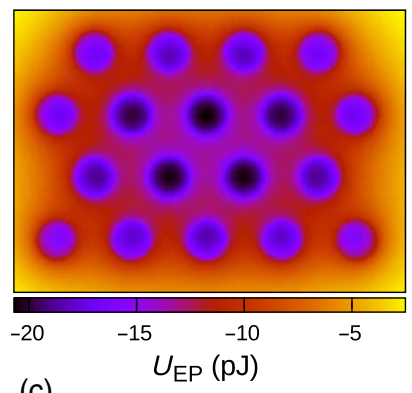

(c)

FIG. 6. Circle mosaic. Patterns made with charged (a) and neutral (b) aluminum nanoparticles and the corresponding electrophoretic (c) and dielectrophoretic (d) potential energies. 
be approximately the same but with larger particle densities at the edges of the motives (edge enhancement).

The present results indicate that, in general, DEP particle patterning gets a more accurate reproduction of the light profile than EP patterning, as the motives are better defined and homogeneous. However, the large number of parameters involved in the process provides the technique with much flexibility. The user has some degrees of freedom to improve or modify the patterning capabilities of their system and to obtain diverse results at will. Some of the most interesting parameters are discussed below.

The first one is the thickness of the photovoltaic crystal. In DEP trapping there is not a great difference in the resulting particle patterns for the typical range of samples with thickness $0.5-1 \mathrm{~mm}$. However, we observe that for EP patterning, the homogeneity of the patterns significantly increases as the crystal thickness decreases. In the top left of Fig. 7(a), a good-quality Al NP particle pattern is shown. It is made with the same experimental parameters as in Fig. 5(a), including the positively charged suspension, except for crystal thickness, which is $0.5 \mathrm{~mm}$ instead of $1 \mathrm{~mm}$. This last pattern is repeated at the bottom of the image for better comparison. It is evident that the pattern made with the thinner crystal is much more homogeneous and that it resembles the light profile much more than the bottom one. The theoretical calculations agree with this result; Fig. 7 also shows the EP potential energy through the same row of squares for 1- and 0.5-mm crystal thickness. As can be seen, in the second case the curve is flatter than in the former one, which means a lower particle gradient through the pattern.

Additional calculations for 0.75 and $2 \mathrm{~mm}$ are made, and they follow this trend: the thinner the crystal, the flatter the potential energy curve. The origin of this dependence is the increasing proximity of the two charged surfaces of the crystal when its thickness decreases, what reduces the total electric field felt by the particle. In addition, in the DEP potential energy curves, the edge effect also decreases

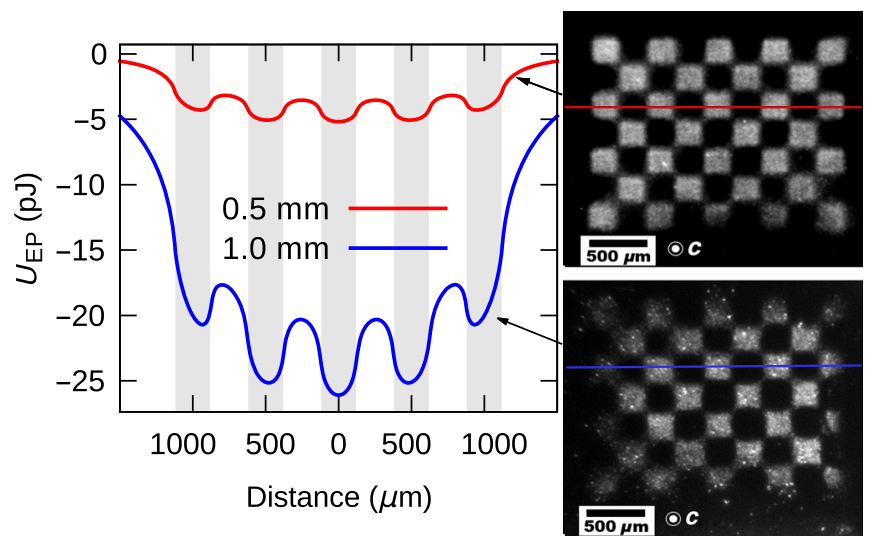

FIG. 7. EP patterning and potential energy curves for two different crystal thicknesses: $0.5 \mathrm{~mm}$ (red line, top) and $1 \mathrm{~mm}$ (blue line, bottom).
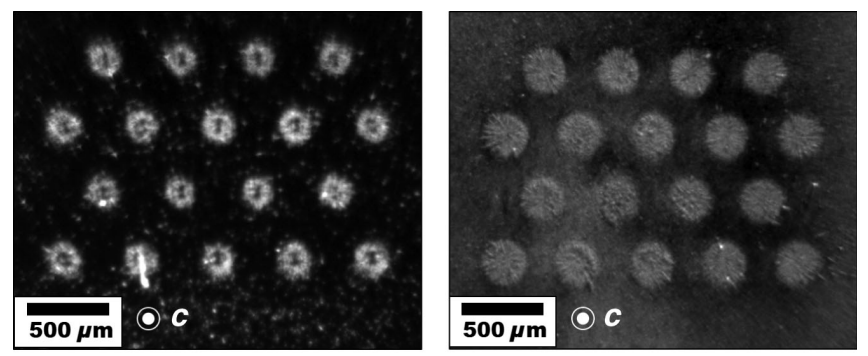

(a)

(b)

FIG. 8. DEP circle patterns made with two different particle concentrations (a) $c=0.01 \mathrm{~g} / \mathrm{L}$ and (b) $c=0.2 \mathrm{~g} / \mathrm{L}$.

with lower crystal thicknesses, although less noticeably. That is the reason why DEP experiments usually show a slight edge enhancement even when thin crystal samples are used.

Two other simple but important parameters are immersion time and suspension concentration. They cannot be addressed independently as the higher the concentration, the lower the immersion time needed to obtain similar patterns. Looking again at Fig. 5(c), the value of the DEP potential energy in the edges of each square is much lower than in the center. This potential distribution produces an increase in the particle density in the edges of each individual square, as shown in the inset of Fig. 5. In the limit in which the particle density or immersion time is low enough, one can obtain DEP patterns similar to the one presented in Fig 8(a), in which even though the illumination is a distribution of solid circles, Al NPs define only a circumference (edge patterning). The experimental parameters of this pattern are the same as in Fig. 5 but with a decrease of the NP concentration from 0.04 to $0.01 \mathrm{~g} / \mathrm{L}$. The opposite case is shown in Fig. 8(b). For this experiment, the concentration increases up to $0.2 \mathrm{~g} / \mathrm{L}$, and as a consequence, the particle density in the center is no longer lower than in the edges but equal to it, although the number of particles in the background also increases, which is usually undesirable.

\section{SUMMARY AND OUTLOOK}

The behavior of charged particles under PV-induced electrophoretic forces, i.e., the manipulation of these particles by PVT, is thoroughly characterized via theoretical calculations and experimental patterns. Moreover, the different features of EP and DEP NP trapping and patterning are analyzed in detail. Charged and neutral aluminum nanoparticles are trapped on the surface of a photovoltaic substrate $\left(\mathrm{Fe}: \mathrm{LiNbO}_{3}\right)$ following the given 2D light patterns. We find that under the influence of EP potentials, NP patterns tend to exhibit radial fading, with larger particle density and pattern definition in the central region with regard to the edges. On the contrary, DEP trapping produces very homogeneous patterns, faithful to the light pattern, although a noticeable edge enhancement of the 
motives is visible, and it tends to present more particles out of place than EP patterning.

These experimental results are discussed and compared with theoretical simulations for the same conditions, showing really good agreement. Although the calculations are based on a previously reported theoretical model that describes DEP trapping [31], the present work extends the applicability of this model to be able to also obtain the EP potentials. In addition, the potential energies' values are calculated, showing that they are much larger than the thermal energy at room temperature, indicating a good stability of the patterns. Despite the model simplicity, it is proven as a useful tool for predicting and understanding all the experimental results.

It is worth mentioning that most of the conclusions presented above are essentially applicable regardless of the method used for obtaining the electric field. In particular, there are related optoelectronic techniques and nonoptical electric deposition techniques on ferroelectric substrates that take advantage of charge distributions similar to the PVT ones for particle arranging. One may point out pyroelectric and ferroelectric domain-based trapping of biological $[36,37]$ and inorganic $[6,38]$ matter and photoinduced $\mathrm{Ag}-\mathrm{NP}$ deposition from $\mathrm{Ag}^{+}$on $\mathrm{Fe}: \mathrm{LiNbO}_{3}$ [39].

Furthermore, the interest and impact of the obtained results is twofold. First, this work provides a characterization of the trapping behavior of charged particles by PVT, a pending issue in the development of this technique. We believe these results will encourage the use of non-neutral objects, as very few studies have been reported so far. As an example, one may consider the controlled deposition in complex shapes of relevant charged biological polymers such as DNA and RNA or other organic compounds as dyes. Second, the comparative analysis of the electrophoretic and dielectrophoretic regimes provides routes for PV patterning optimization when the manipulated nano- or micro-object can be either charged or neutral, as the metal NP shown in this work. In those cases, one may choose which configuration, EP or DEP, is better adapted to the specific needs of their particular application.

\section{ACKNOWLEDGMENTS}

This work is supported by the Spanish Ministerio de Economía y Competitividad under Grant No. MAT201457704-C3. J. F. M.-M. is partially supported by a fellowship of the Universidad Politécnica de Madrid (Grant No. RR01/2016).

[1] D. G. Grier, A revolution in optical manipulation, Nature (London) 424, 810 (2003).

[2] P. Y. Chiou, A. T. Ohta, and M. C. Wu, Massively parallel manipulation of single cells and microparticles using optical images, Nature (London) 436, 370 (2005).
[3] O. M. Marago, P. H. Jones, P. G. Gucciardi, G. Volpe, and A. C. Ferrari, Optical trapping and manipulation of nanostructures, Nat. Nanotechnol. 8, 807 (2013).

[4] S. Grilli, S. Coppola, G. Nasti, V. Vespini, G. Gentile, V. Ambrogi, C. Carfagna, and P. Ferraro, Hybrid ferroelectricpolymer microfluidic device for dielectrophoretic selfassembling of nanoparticles, RSC Adv. 4, 2851 (2014).

[5] C. Sones, I. Katis, B. Mills, M. Feinaeugle, A. Mosayyebi, J. Butement, and R. Eason, Rapid and mask-less laserprocessing technique for the fabrication of microstructures in polydimethylsiloxane, Appl. Surf. Sci. 298, 125 (2014).

[6] A. Gallego, A. García-Cabañes, M. Carrascosa, and L. Arizmendi, Pyroelectric trapping and arrangement of nanoparticles in lithium niobate opposite domain structures, J. Phys. Chem. C 120, 731 (2016).

[7] A. Ashkin, Acceleration and Trapping of Particles by Radiation Pressure, Phys. Rev. Lett. 24, 156 (1970).

[8] M. Carrascosa, A. García-Cabañes, M. Jubera, J. B. Ramiro, and F. Agulló-López, $\mathrm{LiNbO}_{3}$ : A photovoltaic substrate for massive parallel manipulation and patterning of nanoobjects, Appl. Phys. Rev. 2, 040605 (2015).

[9] J. Villarroel, H. Burgos, A. García-Cabañes, M. Carrascosa, A. Blázquez-Castro, and F. Agulló-López, Photovoltaic versus optical tweezers, Opt. Express 19, 24320 (2011).

[10] P. Sturman, Photovoltaic and Photo-Refractive Effects in Noncentrosymmetric Materials, Ferroelectricity and Related Phenomena (Taylor \& Francis, London, 1992).

[11] E. M. de Miguel, J. Limeres, M. Carrascosa, and L. Arizmendi, Study of developing thermal fixed holograms in lithium niobate, J. Opt. Soc. Am. B 17, 1140 (2000).

[12] L. Chen, S. Li, B. Fan, W. Yan, D. Wang, L. Shi, H. Chen, D. Ban, and S. Sun, Dielectrophoretic behaviours of microdroplet sandwiched between LN substrates, Sci. Rep. 6, 29166 (2016).

[13] M. Gazzetto, G. Nava, A. Zaltron, I. Cristiani, C. Sada, and P. Minzioni, Numerical and experimental study of optoelectronic trapping on iron-doped lithium niobate substrate, Crystals 6, 123 (2016).

[14] L. Chen, B. Fan, W. Yan, S. Li, L. Shi, and H. Chen, Photoassisted splitting of dielectric microdroplets in a LN-based sandwich structure, Opt. Lett. 41, 4558 (2016).

[15] L. Miccio, M. Paturzo, A. Finizio, and P. Ferraro, Light induced patterning of poly(dimethylsiloxane) microstructures, Opt. Express 18, 10947 (2010).

[16] I. Elvira, J. F. Muñoz-Martínez, M. Jubera, A. GarcíaCabañes, J. L. Bella, P. Haro-González, M. A. Díaz-García, F. Agulló-López, and M. Carrascosa, Plasmonic enhancement in the fluorescence of organic and biological molecules by photovoltaic tweezing assembly, Adv. Mater. Technol., 1700024 (2017).

[17] A. Blázquez-Castro, J. C. Stockert, B. n. López-Arias, A. Juanrranz, F. Agulló-López, A. García-Cabañes, and M. Carrascosa, Tumour cell death induced by the bulk photovoltaic effect of $\mathrm{LiNbO}_{3}: \mathrm{Fe}$ under visible light irradiation, Photochem. Photobiol. Sci. 10, 956 (2011).

[18] L. Miccio, V. Marchesano, M. Mugnano, S. Grilli, and P. Ferraro, Light induced DEP for immobilizing and orienting Escherichia coli bacteria, Opt. Lasers Eng. 76, 34 (2016). 
[19] M. Jubera, I. Elvira, A. García-Cabañes, J. L. Bella, and M. Carrascosa, Trapping and patterning of biological objects using photovoltaic tweezers, Appl. Phys. Lett. 108, 023703 (2016).

[20] J. F. Muñoz-Martínez, M. Jubera, J. Matarrubia, A. GarcíaCabañes, F. Agulló-López, and M. Carrascosa, Diffractive optical devices produced by light-assisted trapping of nanoparticles, Opt. Lett. 41, 432 (2016).

[21] C. Arregui, J. B. Ramiro, A. Alcázar, A. Méndez, H. Burgos, A. García-Cabañes, and M. Carrascosa, Optoelectronic tweezers under arbitrary illumination patterns: Theoretical simulations and comparison to experiment, Opt. Express 22, 29099 (2014).

[22] H. A. Eggert, F. Y. Kuhnert, K. Buse, J. R. Adleman, and D. Psaltis, Trapping of dielectric particles with light-induced space-charge fields, Appl. Phys. Lett. 90, 241909 (2007).

[23] M. Esseling, F. Holtmann, M. Woerdemann, and C. Denz, Two-dimensional dielectrophoretic particle trapping in a hybrid crystal/PDMS-system, Opt. Express 18, 17404 (2010).

[24] H. Burgos, M. Jubera, J. Villarroel, A. García-Cabañes, F. Agulló-López, and M. Carrascosa, Role of particle anisotropy and deposition method on the patterning of nano-objects by the photovoltaic effect in $\mathrm{LiNbO}_{3}, \mathrm{Opt}$. Mater. 35, 1700 (2013).

[25] J. Matarrubia, A. García-Cabañes, J. Plaza, F. AgullóLópez, and M. Carrascosa, Optimization of particle trapping and patterning via photovoltaic tweezers: Role of light modulation and particle size, J. Phys. D 47, 265101 (2014).

[26] L. Miccio, P. Memmolo, S. Grilli, and P. Ferraro, All-optical microfluidic chips for reconfigurable dielectrophoretic trapping through SLM light induced patterning, Lab Chip 12, 4449 (2012).

[27] J. F. Muñoz-Martínez, I. Elvira, M. Jubera, A. GarcíaCabañes, J. B. Ramiro, C. Arregui, and M. Carrascosa, Efficient photo-induced dielectrophoretic particle trapping on $\mathrm{Fe}: \mathrm{LiNbO}_{3}$ for arbitrary two dimensional patterning, Opt. Mater. Express 5, 1137 (2015).

[28] X. Zhang, J. Wang, B. Tang, X. Tan, R. A. Rupp, L. Pan, Y. Kong, Q. Sun, and J. Xu, Optical trapping and manipulation of metallic micro/nanoparticles via photorefractive crystals, Opt. Express 17, 9981 (2009).
[29] M. Esseling, A. Zaltron, C. Sada, and C. Denz, Charge sensor and particle trap based on z-cut lithium niobate, Appl. Phys. Lett. 103, 061115 (2013).

[30] G. C. L. Wong and L. Pollack, Electrostatics of strongly charged biological polymers: Ion-mediated interactions and self-organization in nucleic acids and proteins, Annu. Rev. Phys. Chem. 61, 171 (2010).

[31] C. Arregui, J. B. Ramiro, A. Alcázar, A. Méndez, J. F. Muñoz-Martínez, and M. Carrascosa, Comparative theoretical analysis between parallel and perpendicular geometries for 2D particle patterning in photovoltaic ferroelectric substrates, J. Eur. Opt. Soc., Rapid Publ. 10, 15026 (2015).

[32] F. Agulló-López, G. Calvo, and M. Carrascosa, in Photorefractive Materials and Their Applications 1, edited by $\mathrm{P}$. Günter and J.-P. Huignard, Springer Series in Optical Sciences Vol. 113 (Springer, New York, 2006), pp. 43-82.

[33] H. A. Pohl, Dielectrophoresis: The Behavior of Neutral Matter in Nonuniform Electric Fields (Cambridge University Press, Cambridge, England, 1978).

[34] P. Mokrý, M. Marvan, and J. Fousek, Patterning of dielectric nanoparticles using dielectrophoretic forces generated by ferroelectric polydomain films, J. Appl. Phys. 107, 094104 (2010).

[35] F. I. Mopsik, Dielectric constant of $n$-Hexane as a function of temperature, pressure and density, J. Res. Natl. Bur. Stand., Sect. A 71A, 287 (1967).

[36] S. Dunn, D. Cullen, E. Abad-Garcia, C. Bertoni, R. Carter, D. Howorth, and R. Whatmore, Using the surface spontaneous depolarization field of ferroelectrics to direct the assembly of virus particles, Appl. Phys. Lett. 85, 3537 (2004).

[37] S. Grilli and P. Ferraro, Dielectrophoretic trapping of suspended particles by selective pyroelectric effect in lithium niobate crystals, Appl. Phys. Lett. 92, 232902 (2008).

[38] P. Mokrý, K. Nováková, M. Marvan, and J. Fousek, Dielectrophoretic forces generated by ferroelectric polydomain films, Phase Transit. 84, 810 (2011).

[39] F. Jia, W. Yan, D. Wang, L. Zhang, L. Shi, A. Lin, G. Liang, M. Li, Y. Zhang, J. Zhang, H. Dong, G. Chen, and H. Chen, Photoinduced Ag-nanoparticle deposition on Fe-doped lithium niobate crystals, Opt. Mater. Express 4, 359 (2014). 